\title{
Application of array CGH on archival formalin-fixed paraffin-embedded tissues including small numbers of microdissected cells
}

\author{
Nicola A Johnson, Rifat A Hamoudi, Koichi Ichimura, Lu Liu, Danita M Pearson, \\ V Peter Collins and Ming-Qing Du
}

Division of Molecular Histopathology, Department of Pathology, University of Cambridge, Cambridge, Cambridgeshire, UK

\begin{abstract}
Array-based comparative genomic hybridisation (aCGH) has diverse applications in cancer gene discovery and translational research. Currently, aCGH is performed primarily using high molecular weight DNA samples and its application to formalin-fixed and paraffin-embedded (FFPE) tissues remains to be established. To explore how aCGH can be reliably applied to archival FFPE tissues and whether it is possible to apply aCGH to small numbers of cells microdissected from FFPE tissue sections, we have systematically performed aCGH on 15 pairs of matched frozen and FFPE astrocytic tumour tissues using a well-established in-house human $1 \mathrm{Mb}$ BAC/PAC genomic array. By spiking tumour DNA with normal DNA, we demonstrated that at least $70 \%$ of tumour DNA was required for reliable aCGH analysis. Using aCGH data from frozen tissue as a reference, it was found that only FFPE astrocytic tumour tissues that supported PCR amplification of $>300$ bp DNA fragment provided high quality, reproducible aCGH data. The presence of necrosis in a tissue specimen had an adverse effect on the quality of aCGH, while fixation in formalin for up to $96 \mathrm{~h}$ of fresh tissue did not appear to affect the quality of the result. As little as $10-20 \mathrm{ng}$ DNA from frozen or FFPE tissues could be readily used for aCGH analysis following whole genome amplification (WGA). Furthermore, as few as $\mathbf{2 0 0 0}$ microdissected cells from haematoxylin-stained slides of archival FFPE tissues could be successfully used for aCGH investigations when WGA was used. By careful assessment of DNA integrity and review of histology, to exclude necrosis and select specimens with a high proportion of tumour cells, it is feasible to preselect archival FFPE tissues adequate for aCGH analysis. With the help of microdissection and WGA, it is also possible to apply aCGH to histologically defined lesions, such as carcinoma in situ.
\end{abstract}

Laboratory Investigation (2006) 86, 968-978. doi:10.1038/labinvest.3700441; published online 5 June 2006

Keywords: array $\mathrm{CGH}$; archival fixed tissue; microdissection; whole genome amplification; astrocytic tumour

Comparative genomic hybridisation (CGH) is a relatively new technology designed to allow high throughput screening of chromosomal gains and losses in diseases, particularly cancer. Traditionally, CGH was carried out by competitive in situ hybridisation of differentially labelled tumour and normal DNA to normal metaphase chromosome spreads. The advent of array-based CGH (aCGH), which uses genome-mapped and sequence-verified genomic clones arrayed on glass slides as the

Correspondence: Professor M-Q Du, PhD, Division of Molecular Histopathology, Department of Pathology, University of Cambridge, Box 231, Level 3 Lab Block, Addenbrooke's Hospital, Hills Road, Cambridge, Cambridgeshire CB2 2QQ, UK.

E-mail: mqd20@cam.ac.uk

Received 16 March 2006; revised and accepted 25 April 2006; published online 5 June 2006 hybridisation target, has radically transformed this technique. aCGH not only confers high resolution but is also exceptionally versatile in its design and application. Essentially, the resolution of aCGH is determined by the size and/or gaps between genomic clones used for array construction. Genomewide BAC arrays at megabase (1 Mb) or submegabase $(100 \mathrm{~kb})$ size are now available for screening of genomic gains/losses. ${ }^{1,2}$ Genomic copy number changes can be further characterised by use of chromosome-specific or customised tiled arrays, ${ }^{3,4}$ which can be tailor made at any desired resolution. In addition, chromosome-specific tiled arrays can be used for characterisation of chromosomal translocation breakpoints when the derivative chromosome is isolated and used as the source of DNA. ${ }^{5}$ Clearly, aCGH has immense potential in disease gene discovery and translational research. 
aCGH analyses were initially developed using high molecular weight DNA samples from fresh/ frozen tissues or cells. The use of formalin-fixed and paraffin-embedded (FFPE) tissues as a source of DNA has many advantages. Such tissues are available as a huge resource, with detailed histological and phenotypic characterisation and valuable clinical-pathological follow-up data. It would be immensely beneficial if aCGH could be applied to such materials. So far, a few studies have used FFPE-derived DNA for aCGH, ${ }^{6-9}$ and it may be realistic to use small amounts of DNA extracted from whole tissue sections of frozen or FFPE specimens for aCGH following whole genome amplification (WGA). ${ }^{10-12}$ Nonetheless, several critical issues remain to be investigated. Firstly, because DNA quality from FFPE tissues is variable, is it possible to reliably identify suitable archival FFPE tissues for aCGH? Secondly, can aCGH be applied to small numbers of cells microdissected from archival FFPE tissue samples? Thirdly, is it possible to distinguish true tumour-derived alterations from experimental artefacts? To address these issues, we have systematically performed aCGH on 15 pairs of matched frozen and FFPE astrocytic tumour tissues and developed a practical protocol which enables preselection of archival FFPE cases adequate for aCGH analysis and the successful application of the technique to DNA samples extracted from as few as 2000 microdissected cells.

\section{Materials and methods}

\section{Tumour Tissues and Fixation}

The study was based on 15 pairs of matched freshfrozen and FFPE astrocytic tumour tissues (11 glioblastoma xenografts archived for 6 years, four primary glioblastomas and one primary anaplastic astrocytoma archived for 15 years). Diagnostic information, age and sex of patients, including parental tumours of xenograft samples included in the study, are included in Supplemental Table 1. All FFPE tissues were fixed overnight in $10 \%$ buffered formalin, routinely processed and paraffin embedded. Use of these archival tissues for research was approved by the Ethics Committee of the Karolinska Hospital and Cambridge Local Research Ethics Committee.

In addition, to examine the effect of tissue fixation on aCGH, a glioblastoma xenograft tissue sample, previously archived at $-80^{\circ} \mathrm{C}$ for 6 years, was divided into five equal pieces, fixed in $10 \%$ buffered formalin at room temperature for various times (12, $24,48,72$ and $96 \mathrm{~h}$ ), then routinely processed and paraffin embedded.

\section{Microdissection and DNA Extraction}

DNA was extracted from fresh-frozen tumour tissues and blood samples as previously described. ${ }^{13}$
FFPE tissue sections were dewaxed twice in xylene, washed in $100 \%$ ethanol, and digested overnight with $1 \mathrm{mg} / \mathrm{ml}$ proteinase K (Qiagen, Valencia, CA, USA) at $56^{\circ} \mathrm{C}$ in a $200 \mu \mathrm{l}$ reaction mixture containing $30 \mathrm{mM}$ Tris-Cl (pH 8.0), $10 \mathrm{mM}$ EDTA and $1 \%$ sodium dodecyl sulphate. The sample was then mixed with an equal volume of phenol-chloroformisoamyl (25:24:1) (pH 7.9) (Ambion, TX, USA) and centrifuged through a phase-lock heavy gel (Eppendorf AG, Hamburg, Germany). DNA was ethanol precipitated overnight at $-20^{\circ} \mathrm{C}$ and dissolved in $20 \mu \mathrm{l}$ of $10 \mathrm{mM}$ Tris/ $1 \mathrm{mM}$ EDTA.

Crude microdissection was performed on freshly prepared haematoxylin-stained slides to avoid necrotic areas or to isolate various numbers of tumour cells. Microdissected cells were similarly digested with proteinase $\mathrm{K}$ in a $25 \mu \mathrm{l}$ reaction mixture as above and DNA was purified using a DNA microkit (Qiagen).

DNA concentration was determined using PicoGreen $^{\text {TM }}$ (Molecular Probes, Eugene, OR, USA).

\section{Assessment of DNA Integrity}

The integrity of DNA samples from FFPE tissue was assessed by PCR of variable sized DNA fragments $(100,200,300,400$ and $600 \mathrm{bp})$ in separate reactions using $10 \mathrm{ng}$ of template DNA as described previously. ${ }^{14}$ PCR products were analysed by electrophoresis on $2 \%$ agarose gels.

\section{WGA}

Various amounts (10-20 ng) of DNA from freshfrozen or FFPE tissue was amplified using GenomePlex $^{\mathrm{TM}}$ WGA kit (Rubicon Genomics, Ann Arbor, MI, USA). Amplified genomic DNA was purified using DNA Clean and Concentrator (Zymo Research, Orange, CA, USA) and quantified using PicoGreen.

\section{Array CGH}

CGH arrays were constructed in-house based on protocols used by the Wellcome Trust Sanger Institute, Cambridge, $\mathrm{UK}^{2}$ and comprised 3038 analysable BAC clones spaced at approximately $1 \mathrm{Mb}$ intervals across the whole genome. Construction and validation of the $1 \mathrm{Mb}$ genomic array has been previously described. ${ }^{2,15}$ Briefly, clone DNA was extracted and amplified using three DOP primers which were subsequently mixed and amplified using a $5^{\prime}$-amine modified universal primer. Amine-linked PCR products were arrayed onto amine-binding slides (CodeLink, Amersham Biosciences, Little Chalfont, Buckinghamshire, UK) in duplicate. Each array was composed of 24 blocks; Drosophila clones and clones from individual chromosomes were evenly distributed throughout all blocks.

Labelling of test (tumour) and reference (control) DNA, array hybridisation and washing were carried 
out essentially as previously described., ${ }^{3,15}$ Briefly, $400 \mathrm{ng}$ of test (tumour) and reference DNA were labelled using a Bioprime Labelling Kit (Invitrogen, Carlsbad, CA, USA) with a modified dNTP reaction mixture. Test DNA was hybridised with sex-mismatched reference DNA from samples of pooled blood from 20 normal males or 20 normal females. Labelled and purified test and reference DNA were pooled and co-precipitated with $45 \mu \mathrm{g}$ Cot-1 DNA (Roche Diagnostics, Mannheim, Germany) and $400 \mu \mathrm{g}$ herring sperm DNA (Sigma-Aldrich, St Louis, MO, USA). The precipitated DNA was dissolved in hybridisation buffer and hybridised to an array that had been prehybridised with $80 \mu \mathrm{g}$ Cot-1 DNA and $400 \mu \mathrm{g}$ herring sperm DNA for $2 \mathrm{~h}$. Arrays were allowed to hybridise overnight at $37^{\circ} \mathrm{C}$ then washed as described. ${ }^{3}$

\section{aCGH Data Analysis}

aCGH slides were scanned using an Axon 4100A scanner (Axon Instruments, Burlingame, CA, USA). Scanned images were quantified using GenePix Pro 5.1 software (Axon Instruments) and primary data analysis and normalisation carried out using Microsoft Excel. ${ }^{3}$ An average between the duplicate spotted BACs was calculated and results were analysed using plots of $\log _{2}$-transformed normalised Cy5:Cy3 intensity ratios against clone position.

The mean and standard deviation (s.d.) used as the threshold value for identifying genomic gains and losses were determined from four normal male/ female hybridisations. Mean \pm 3 s.d. (equivalent to $\log _{2}$ value \pm 0.19 ) was used as the threshold for aCGH using DNA from frozen tissue. Systematic analysis of the mean \pm various s.d. $(3,3.2,3.4,3.6$, $3.8,4)$ on nine good quality aCGH FFPE astrocytic tumour samples (five xenograft and four primary) data sets were used to determine the threshold value for aCGH with and without WGA. Altered clones were identified and percentages of concordance and nonconcordance with those derived from the corresponding frozen tissue were calculated. The mean \pm 3.2 s.d. $\left(\log _{2}\right.$ value \pm 0.20$)$ and mean \pm 3.4 s.d. $(\log 2$ value +0.22$)$ was chosen as the cutoff for FFPE tissue and FFPE with WGA, respectively. The three cutoff values chosen allowed confident detection of single copy gain or loss since the imbalance involving single copy change as demonstrated by chromosome X clones between normal male/female hybridisations typically showed $\log _{2}$ ratio changes in the range of 0.55-0.67. The suitability of these cutoff values was further verified by detection of single copy gains/losses even when the percentage of tumour cells was at $75 \%$ (detailed in Results).

Alterations that involve consecutive clones are likely to represent true genomic alterations which commonly affect a relatively large chromosomal region. ${ }^{16}$ However, it is more difficult to distinguish changes affecting single clones that may result from random or experimental variations, such as mismapped clones or crosshybridisation due to repetitive sequences. To test this, we compared the reproducibility of aCGH changes in four replicate aCGH hybridisations, using a DNA sample from frozen xenograft tissue.

\section{Statistics}

Comparison of the aCGH data of the same specimen among different experimental conditions (Pearson's correlation test) and unsupervised hierarchical clustering was carried out using stats package in $\mathrm{R}$ version 2.1.1 ${ }^{17}$ (http://www.R-project.org).

\section{Results}

\section{Reproducibility of aCGH Changes Involving Consecutive Clones or Single Clones}

To compare the reproducibility of aCGH changes involving consecutive or single clones, we performed four independent aCGH analyses using the same DNA sample from a frozen xenograft tissue sample. The concordant clones from all four independent experiments were used as reference to assess the reproducibility of each independent hybridisation. As expected, aCGH changes affecting two or more consecutive clones were highly reproducible (mean 88\%), while those involving single clones were not (mean 16\%) (Table 1). Similar results were seen when using DNA from frozen and FFPE tissues of the same cases (Supplemental Table 2). This finding indicated that most of the changes affecting single clones were likely to be the result of experimental artefacts. For this reason, assessment of concordance between frozen and FFPE tissue under various experimental conditions focused on changes involving two or more consecutive clones.

\section{aCGH: Minimum Proportion of Tumour DNA Required for Reliable aCGH Analysis}

Tumour tissues invariably contain non-neoplastic cells. To examine the minimal proportion of tumour DNA required for reliable aCGH analysis, a DNA sample with known homozygous and hemizygous deletions from one frozen glioblastoma xenograft (X1) was mixed with various amounts of nontumour DNA and then subjected to aCGH analyses. The ability of the aCGH technology to detect genomic gains/losses was shown to critically depend on the proportion of tumour DNA (Figure 1). When tumour DNA was $\leq 50 \%$, both chromosomal gain and loss (including those affecting relatively large regions) became difficult to recognise (Figure 1) and their concordance with aCGH data from $100 \%$ tumour DNA was poor. Nonetheless, reliable CGH results 
Table 1 aCGH using DNA from frozen tissue: reproducibility between consecutive and single clones affected

Total no. of clones affected (cutoff value mean \pm 3 s.d.)
Consecutive clones affected

\begin{tabular}{cc}
\hline $\begin{array}{c}\text { No. clones } \\
\text { affected }\end{array}$ & $\begin{array}{r}\text { Percentage } \\
\text { reproducibility }\end{array}$
\end{tabular}

$(\%)$
Single clones affected

$\begin{array}{cc}\begin{array}{c}\text { No. clones } \\ \text { affected }\end{array} & \text { Percentage } \\ \text { reproducibility }\end{array}$

$(\%)$

\begin{tabular}{|c|c|c|c|c|c|}
\hline Reference $^{\mathrm{a}}$ & 292 & 283 & & 9 & \\
\hline A951 replicate 1 & 372 & 338 & 84 & 34 & 26 \\
\hline A951 replicate 2 & 476 & 359 & 79 & 117 & 8 \\
\hline A951 replicate 3 & 363 & 305 & 93 & 58 & 16 \\
\hline A951 replicate 4 & 353 & 291 & 97 & 62 & 15 \\
\hline Mean & 391 & 323 & 88 & 68 & 16 \\
\hline
\end{tabular}

${ }^{\mathrm{a}}$ Reference was calculated by extracting concordant consecutive and single clones in all four individual aCGH experiments, as these are less likely to be due to experimental artefacts.

Effect of proportion of tumor derived DNA on aCGH.

\begin{tabular}{|c|c|c|c|c|c|}
\hline \multirow[b]{3}{*}{$100 \%$ tumor DNA } & \multirow{3}{*}{$\begin{array}{c}\text { Total no. clones } \\
\text { affected }\end{array}$} & \multicolumn{4}{|c|}{ Consecutive clones affected } \\
\hline & & \multicolumn{2}{|c|}{ No. clones affected } & \multicolumn{2}{|c|}{$\begin{array}{c}\text { No. clones concordant with those from } \\
100 \% \text { tumor DNA and percentage of } \\
\text { successful detection }\end{array}$} \\
\hline & & 389 & $92 \%$ & & \\
\hline $75 \%$ tumor DNA & 390 & 372 & $96 \%$ & 372 & $96 \%$ \\
\hline $50 \%$ tumor DNA & 177 & 145 & $75 \%$ & 145 & $37 \%$ \\
\hline
\end{tabular}
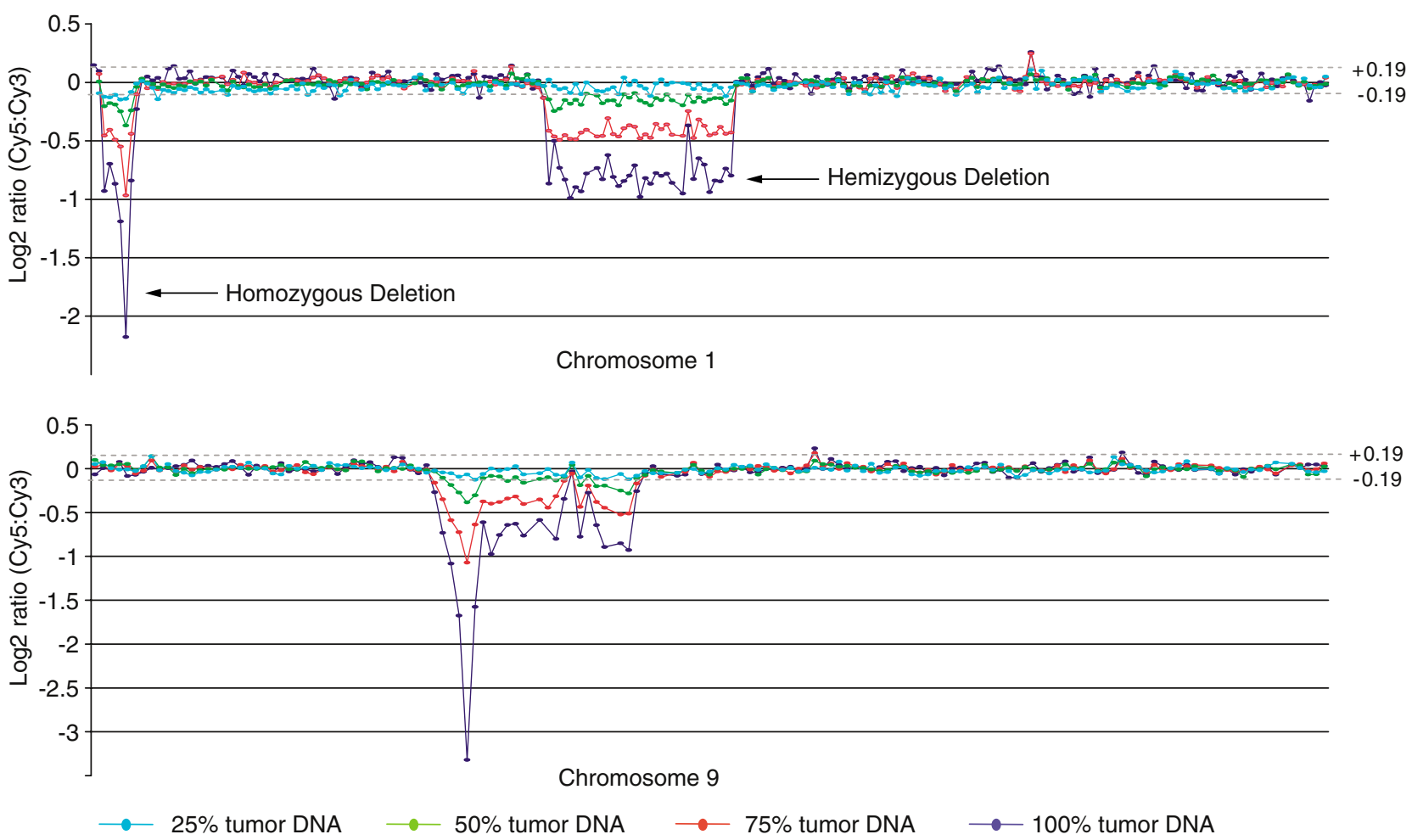

Figure 1 Sensitivity of aCGH. High molecular DNA from a frozen glioblastoma xenograft was diluted with DNA from normal blood samples at various ratios and subjected to $1 \mathrm{Mb}$ aCGH analysis. The $\log _{2}$ ratio of tumour/reference fluorescence is shown for chromosomes 1 and 9 . Clones are arranged in genomic order from pter to qter. The data indicates that at least $75 \%$ of tumour DNA is required for reliable aCGH analysis. 
Table 2 aCGH using DNA from FFPE tissue: minimum requirement for DNA integrity, DNA quantity or number of cells

\begin{tabular}{llll}
\hline Sample & $\begin{array}{c}\text { Maximum DNA } \\
\text { fragment }(\mathrm{bp}) \\
\text { amplified }\end{array}$ & $\begin{array}{l}\text { Quality of aCGH } \\
\text { by visual } \\
\text { inspection }\end{array}$ & Pearson's correlation to aCGH data from corresponding \\
frozen tissue
\end{tabular}

${ }^{\mathrm{a}}$ Poor correlation was due to the presence of $>20 \%$ necrosis.

could be obtained when the proportion of tumour DNA was $\geq 75 \%$.

\section{aCGH Using DNA from FFPE Tissues: Effect of DNA Integrity and Necrosis}

To examine to what extent archival FFPE tissues could be reliably used for aCGH analysis, DNA samples from whole tissue sections of archival FFPE glioblastoma xenografts were subjected to aCGH analysis and the resulting data compared to that from corresponding frozen tissues. The quality of aCGH data from FFPE tissues was then compared to the integrity of DNA samples.

Of the 11 FFPE glioblastoma xenografts with matched frozen tissues, sufficient DNA for aCGH analysis could be extracted from nine cases. All nine cases yielded a quality DNA sample (as assessed spectrophotometrically) with a similar smear pattern on $1 \%$ agarose gels, but gave rise to dramatically variable aCGH data quality when compared to that from the corresponding frozen tissue (Table 2, Figure 2). In four cases (X1, X3-5), a good correlation $(r=0.83-0.98)$ between data generated from FFPE and frozen tissues was obtained and 79-98\% of CGH changes involving two or more consecutive clones identified from DNA samples of frozen tissues were seen when using DNA samples from FFPE tissue (Table 3). It has to be stated that the true reproducibility of aCGH on FFPE tissue was most likely underestimated as $12 \%$ of aCGH changes involving two or more consecutive clones seen from frozen tissues were not reproducible (Table 1). In the remaining five cases, the quality of aCGH data from FFPE tissues was poor (Table 2).

The quality of aCGH from FFPE tissues corresponded well to the integrity of the DNA sample as shown by PCR of variable sized gene fragments. All four FFPE DNA samples that yielded representative aCGH data also supported amplification of a DNA fragment $>300 \mathrm{bp}$. Only one FFPE tissue specimens (X2) that produced poor quality data yielded a DNA sample that showed PCR amplification of a DNA fragment $>300 \mathrm{bp}$ (Table 2).

To examine why specimen $\mathrm{X} 2$, with relatively good DNA integrity, failed aCGH analysis, we reviewed the histology. X2 contained a prominent necrotic area $(\sim 20 \%)$, while all other cases displayed minimal necrotic regions $(<5 \%)$ or no necrosis. To test whether the presence of necrosis had an adverse effect on aCGH, we repeated aCGH using a DNA sample prepared from the microdissected 'viable' tumour cells, which resulted in highquality aCGH results (Supplemental Figure 1), comparable to those from the corresponding frozen tissue $(r=0.94)$.

\section{aCGH Using DNA from FFPE Tissues: Effect of Formalin Fixation and Storage Time}

To examine the effect of the length of formalin fixation on aCGH, tissue samples from one xenograft 

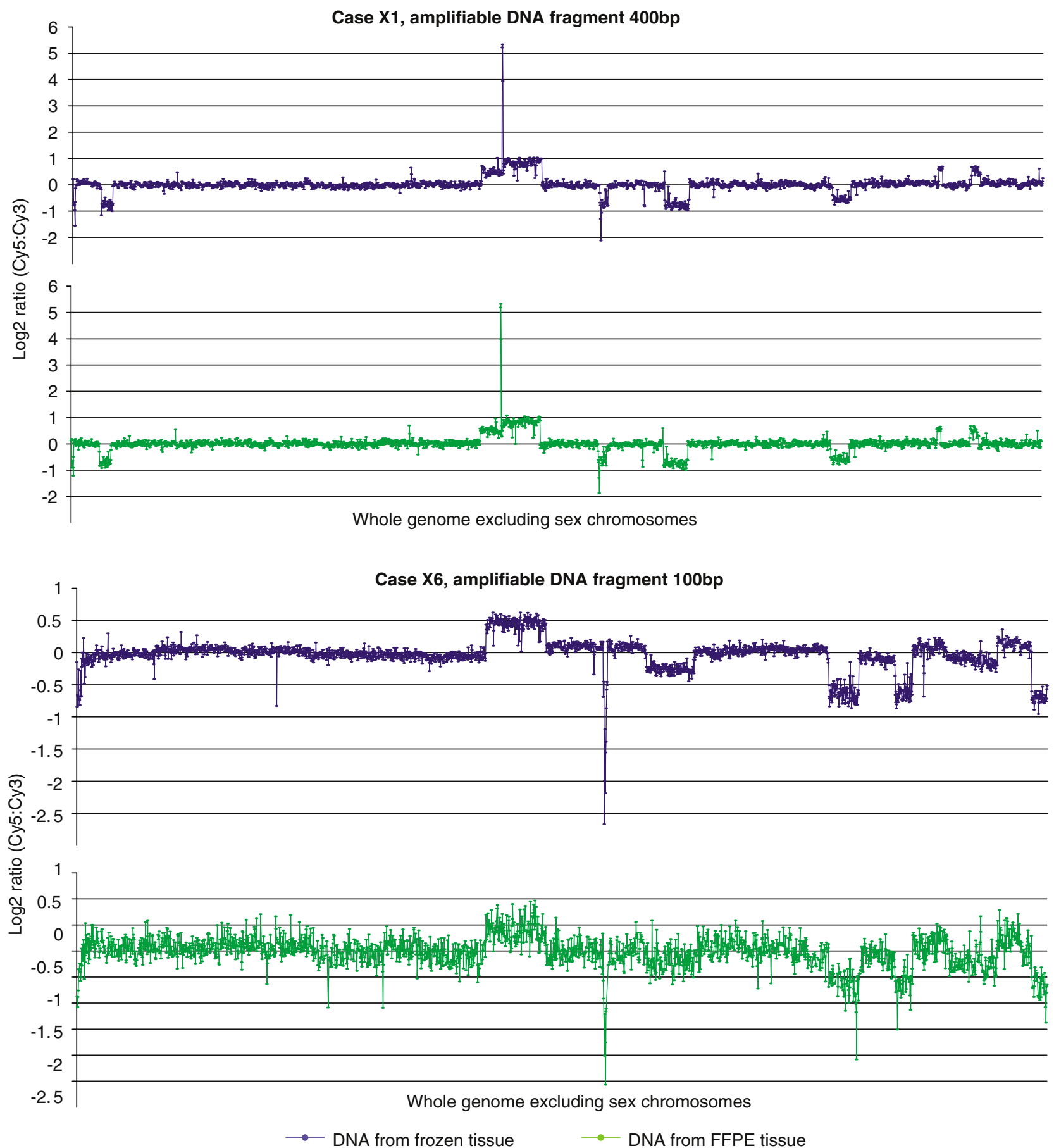

Figure 2 Effect of DNA integrity on aCGH analysis. DNA samples from matched frozen and FFPE glioblastoma xenograft are subjected to $1 \mathrm{Mb}$ aCGH analysis. The $\log _{2}$ ratio of tumour/reference fluorescence is shown for all BAC clones, which are arranged in genomic order from 1pter to 22qter. In case X1, the DNA sample from the FFPE glioblastoma xenograft is of good integrity (amplifiable for $400 \mathrm{bp}$ fragment) and shows a highly reproducible CGH profile as compared with the DNA from corresponding frozen tissue. Whereas in case $\mathrm{X6}$, the DNA sample from the FFPE glioblastoma xenograft is of poor integrity (only amplifiable for $100 \mathrm{bp}$ fragment), displays a CGH profile with high background noise and fails to demonstrate the genomic aberrations seen from the DNA sample from the corresponding frozen tissue.

were fixed in $10 \%$ buffered formalin for various times, routinely processed and paraffin embedded and subjected to aCGH. The aCGH data obtained was compared with that from the corresponding frozen tissue (Supplemental Table 3). The results showed that formalin fixation up to $96 \mathrm{~h}$ did not have any major adverse effect on the quality of aCGH. DNA samples prepared from these FFPE 
Table 3 Correlation of aCGH between DNA samples from frozen tissues, FFPE tissue and small numbers of cells microdissected from FFPE tissue sections

\begin{tabular}{|c|c|c|c|c|c|c|c|c|c|c|c|}
\hline & \multicolumn{2}{|c|}{$\begin{array}{l}\text { DNA from frozen tissue } \\
\text { (cutoff value mean } \pm 3 \text { s.d.) }\end{array}$} & \multicolumn{3}{|c|}{$\begin{array}{l}400 \text { ng DNA from FFPE tissue } \\
\text { (cutoff value mean } \pm 3.2 \text { s.d.) }\end{array}$} & \multicolumn{3}{|c|}{$\begin{array}{l}20 \text { ng DNA from FFPE tissue with WGA } \\
\text { (cutoff value mean } \pm 3.4 \text { s.d.) }\end{array}$} & \multicolumn{3}{|c|}{$\begin{array}{l}2000 \text { cells from FFPE tissue with WGA } \\
\text { (cutoff value mean } \pm 3.4 \text { s.d.) }\end{array}$} \\
\hline & \multirow{2}{*}{$\begin{array}{l}\text { Total no. of } \\
\text { clones } \\
\text { affected }\end{array}$} & \multirow{2}{*}{$\begin{array}{c}\text { No. of } \\
\text { consecutive } \\
\text { clones } \\
\text { affected }\end{array}$} & \multirow{2}{*}{$\begin{array}{l}\text { Total no. of } \\
\text { clones } \\
\text { affected }\end{array}$} & \multicolumn{2}{|c|}{ Consecutive clones affected } & \multirow{2}{*}{$\begin{array}{l}\text { Total no. of } \\
\text { clones } \\
\text { affected }\end{array}$} & \multicolumn{2}{|c|}{ Consecutive clones affected } & \multirow{2}{*}{$\begin{array}{l}\text { Total no. of } \\
\text { clones } \\
\text { affected }\end{array}$} & \multicolumn{2}{|c|}{ Consecutive clones affected } \\
\hline & & & & $\begin{array}{l}\text { No. of } \\
\text { clones } \\
\text { affected }\end{array}$ & $\begin{array}{l}\text { No. of clones } \\
\text { concordant } \\
\text { with those from the } \\
\text { corresponding frozen } \\
\text { tissue and } \\
\text { percentage } \\
\text { of successful } \\
\text { detection }\end{array}$ & & $\begin{array}{l}\text { No. of clones } \\
\text { affected }\end{array}$ & $\begin{array}{c}\text { No. of clones } \\
\text { concordant } \\
\text { with those from the } \\
\text { corresponding frozen } \\
\text { tissue and } \\
\text { percentage } \\
\text { of successful } \\
\text { detection }\end{array}$ & & $\begin{array}{l}\text { No. of clones } \\
\text { affected }\end{array}$ & $\begin{array}{c}\text { No. of clones } \\
\text { concordant } \\
\text { with those from the } \\
\text { corresponding frozen } \\
\text { tissue and } \\
\text { percentage } \\
\text { of successful } \\
\text { detection }\end{array}$ \\
\hline $\mathrm{X} 1$ & 480 & $408(85 \%)$ & 458 & $405(88 \%)$ & $399(98 \%)$ & 486 & $396(81 \%)$ & $386(95 \%)$ & 743 & $446(60 \%)$ & $334(82 \%)$ \\
\hline $\mathrm{X} 2$ & 398 & $376(94 \%)$ & 441 & $394(89 \%)$ & $368(98 \%)$ & 588 & $439(75 \%)$ & $370(98 \%)$ & 519 & $398(77 \%)$ & $354(94 \%)$ \\
\hline X3 & 947 & $765(81 \%)$ & 1338 & $1117(83 \%)$ & $637(83 \%)$ & 1212 & $951(78 \%)$ & $618(81 \%)$ & 1215 & 939 (77\%) & $642(84 \%)$ \\
\hline $\mathrm{X} 4$ & 761 & $664(87 \%)$ & 1011 & $771(76 \%)$ & $550(79 \%)$ & 1014 & $742(73 \%)$ & $503(76 \%)$ & & & \\
\hline X5 & 1578 & $1373(87 \%)$ & 1702 & $1509(89 \%)$ & $1155(85 \%)$ & 1563 & $1276(82 \%)$ & $1097(80 \%)$ & & & \\
\hline P1 & 779 & $608(78 \%)$ & 1029 & $745(72 \%)$ & $423(70 \%)$ & & & & & & \\
\hline $\mathrm{P} 2$ & 410 & $401(98 \%)$ & 805 & $580(72 \%)$ & $358(90 \%)$ & & & & 661 & 447 (68\%) & 369 (92\%) \\
\hline P3 & 783 & $743(95 \%)$ & 958 & $726(76 \%)$ & $523(80 \%)$ & & & & & & \\
\hline $\mathrm{P} 4$ & 1048 & $888(85 \%)$ & 1504 & $1265(84 \%)$ & $717(82 \%)$ & & & & & & \\
\hline Mean & 798 & $692(88 \%)$ & 1027 & $835(81 \%)$ & $570(85 \%)$ & 973 & $761(78 \%)$ & $595(86 \%)$ & 785 & $558(70 \%)$ & $425(88 \%)$ \\
\hline
\end{tabular}


specimens showed PCR amplification of a $400 \mathrm{bp}$ DNA fragment and a good correlation to aCGH results using DNA from the corresponding frozen tissue.

To further examine the effect of storage time on aCGH, we performed aCGH on four archival FFPE primary astrocytic tumours that had been stored for 15 years, and compared the data to the corresponding frozen tumours. A good correlation between
FFPE and frozen tissues was seen in each case (Tables 2 and 3 ).

\section{aCGH Using Minimum Amount of DNA}

To explore the potential applications of aCGH to small tissue biopsies and microscopic lesions, we investigated the minimum amount of DNA required for aCGH analysis. We first tested this by using DNA
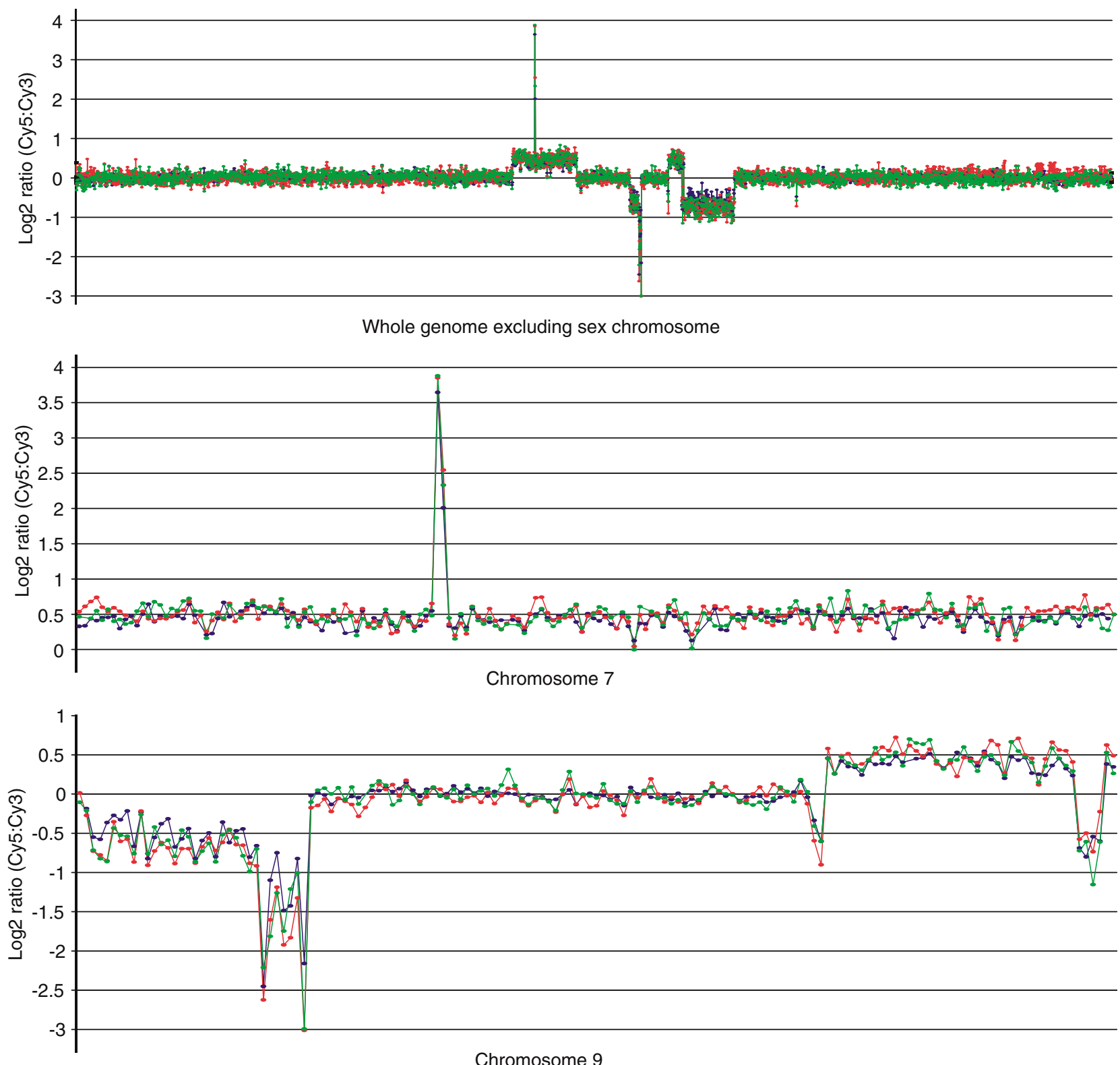

Chromosome 9

- DNA from frozen tissue $\quad \longrightarrow$ DNA from FFPE tissue $\quad \longrightarrow 2.000$ cells from a FFPE tissue section

Figure 3 aCGH using a minimum amount of DNA and $\sim 2000$ cells microdissected from a FFPE glioblastoma xenograft tissue slide. In case X2, 20 ng DNA from the FFPE glioblastoma xenograft and the DNA sample prepared from 2000 cells microdissected from a haematoxylin-stained FFPE xenograft tissue slide are amplified using GenomePlex ${ }^{\mathrm{TM}}$ WGA kit and subjected to aCGH analysis. The $\log _{2}$ ratio of tumour/reference fluorescence is shown for all BAC clones, which are arranged in genomic order from 1pter to $22 \mathrm{qter}$ and highresolution correlation is also shown for chromosomes 7 and 9. The data shows a highly reproducible CGH profile from 20 ng DNA from the FFPE xenograft tissue and the DNA sample from 2000 cells microdissected from a FFPE xenograft tissue slide as compared with DNA sample from the corresponding frozen tumour tissue. 
samples from the frozen glioblastoma xenografts. We performed WGA using the GenomePlex ${ }^{\mathrm{TM}}$ WGA kit using various amounts $(10,20$ and $50 \mathrm{ng})$ of DNA from frozen tissues and found that the minimum amount of DNA required for reliable WGA was $10 \mathrm{ng}$, as recommended by the manufacturer (Rubicon Genomics). DNA from case X2 was amplified using WGA kit and subjected to aCGH. Correlation with data from nonamplified DNA of the same source was high $(r=0.99)$ and no chromosome or gene-specific bias could be observed from the linear plots.

We next tested the minimum amount of DNA from FFPE tissue required for representative aCGH. This was carried out using five xenograft specimens that showed good quality aCGH data from nonamplified DNA samples. In each case, representative data were obtained from a $20 \mathrm{ng}$ DNA template (as recommended by the manufacturer), as judged by comparison with data from the corresponding frozen tissue (Tables 2 and 3, Figure 3).

\section{aCGH Using Minimum Number of Cells Microdissected from FFPE Tissue Sections}

To investigate whether aCGH could be applied to small numbers of cells microdissected from FFPE tissue sections, we microdissected and extracted DNA from various numbers $(10000,5000,2000$ and 1000 cells) of cells from haematoxylin-stained tissue sections, avoiding necrotic regions. Representative aCGH data were obtained from DNA samples extracted from 2000 cells or above, as judged by comparison with data from the corresponding frozen tissue (Tables 2 and 3, Figure 3).

\section{Clustering of aCGH Data from Frozen, FFPE Tissue and Microdissected Cells}

Unsupervised hierarchical clustering analysis showed that all data sets from the same specimen clustered together (Figure 4). This further demonstrated the similarity, thus reproducibility, of aCGH data between frozen, FFPE tissues and microdissected cells.

\section{Discussion}

Archival FFPE tissues represent a rich resource of well-characterised pathological specimens for genetic study of human disease, particularly cancer. By systematic investigations of matched frozen and FFPE astrocytic tumour tissues, we developed a

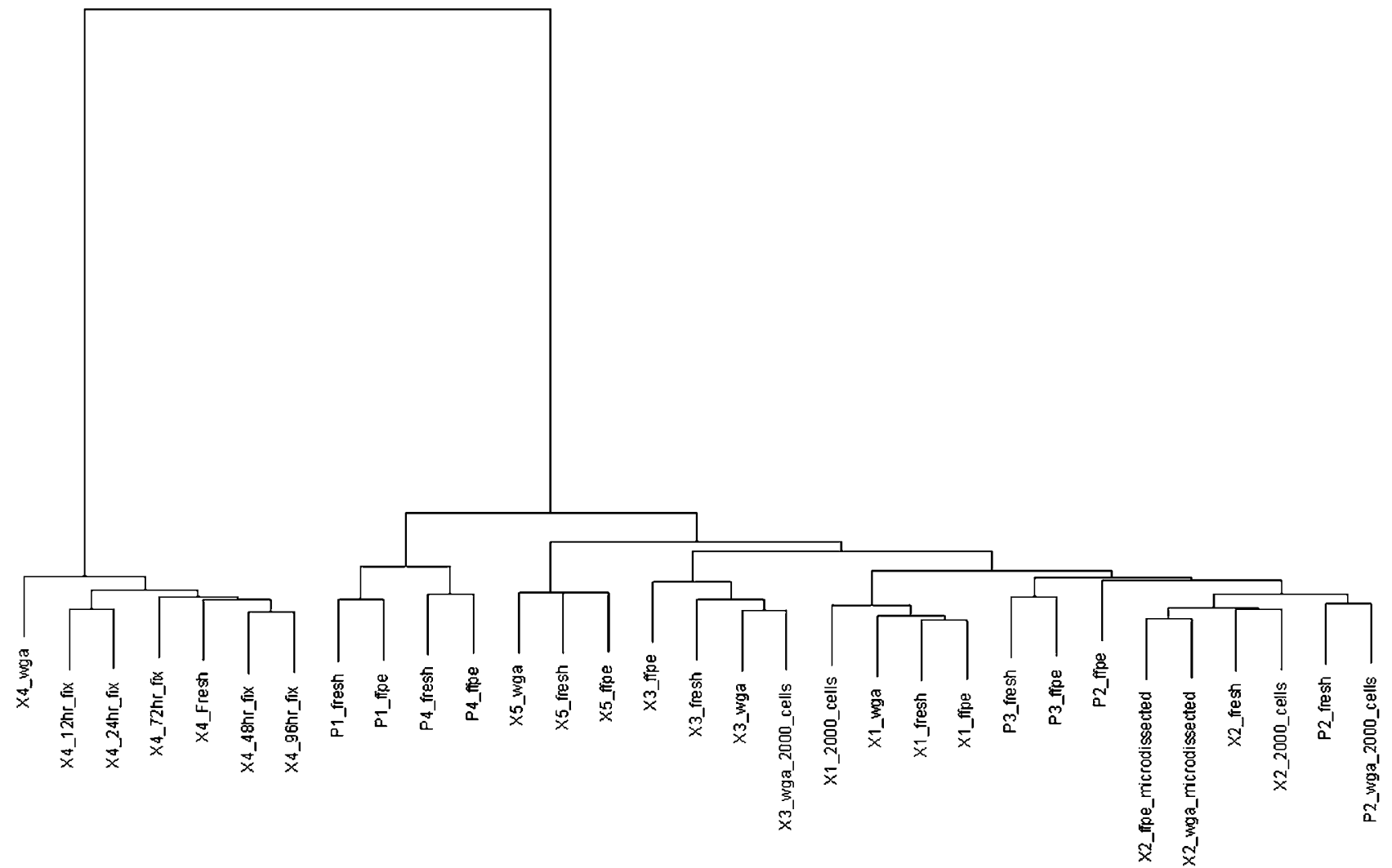

Figure 4 Unsupervised hierarchical clustering. The dendogram demonstrates that aCGH data generated from the same tissue source is clustered together irrespective of whether DNA used is from frozen or FFPE tissue, or amplified from small quantities of DNA or cells. Xenograft sample X2 and patient sample were not derived from the same source but produced a visibly similar aCGH profile. wga, whole genome amplification; 12-96h_fix denotes hours of fixation in formalin; ffpe, formalin-fixed paraffin -embedded; X, glioblastoma xenograft; P, primary astrocytic tumour. 
practical protocol to apply archival FFPE tissues to aCGH. As the quality of aCGH critically depends on the integrity of DNA samples, it is possible to predict whether an archival FFPE tissue specimen is suitable for aCGH analysis when extracted DNA supports PCR amplification of $>300 \mathrm{bp}$ gene fragments. PCR-based DNA integrity assessment is superior to direct examination of the DNA sample on agarose gels, as it is more objective and uses only a minute amount of DNA. In our experience, direct analysis of DNA samples on agarose gels did not offer any value in the prediction of the suitability of a FFPE tissue sample for aCGH.

In general, aCGH alterations involving consecutive BAC clones are easily recognisable and most likely derive from tumour cells rather than experimental artefacts. However, interpretation of aCGH changes affecting single clones is difficult and these sporadic changes occur when using DNA samples from both frozen and FFPE tissues, particularly the latter. In theory, alterations as a result of true genomic events should be reproducible and those highly associated with tumorigenesis and progression are likely recurrent. Conversely, changes due to experimental artefacts are largely random, although those resulting from mismapped clones or crosshybridisation of repetitive sequences may be reproducible. Such assumptions were well supported by our experimental data, demonstrating that aCGH changes involving two or more consecutive clones were highly reproducible, while those involving single sporadic clones were not. In view of this and the vast amount of data potentially derived from an aCGH study, it is pertinent to focus on alterations involving consecutive clones for any follow-up investigations. Nonetheless, genomic amplification/deletion occasionally involves a single gene locus: gene amplification can be readily identified by visual inspection of the aCGH profile, while deletion, if recurrent, should not be neglected.

One of the other major factors affecting the quality of aCGH was the presence of necrosis in tissue specimens. It is likely that the adverse effect of necrosis on aCGH is due to DNA degradation of necrotic cells. However, such DNA degradation contributed by necrosis could not be revealed by PCR-based assessment of DNA integrity, as it would be masked by the relatively intact DNA from 'viable' cells. However, necrosis is easily identified by histological review and its adverse effect on aCGH can be eliminated by microdissection.

In line with loss of heterozygosity analysis, sensitivity of aCGH in detection of DNA copy number changes heavily depends on the proportion of tumour cells in a tissue specimen. ${ }^{18}$ By spiking xenograft tumour DNA with normal DNA, we showed that at least $70 \%$ tumour cells are required for reliable aCGH analysis. Together with the adverse effect of necrosis, this emphasises the importance of routine histological review of tissue specimens selected for aCGH.
With adequate quality control, it was also possible to apply aCGH to small numbers of cells from FFPE tissue sections with a combination of microdissection and WGA. As shown in the present study, we were able to use as few as 2000 cells microdissected from haematoxylin-stained tissue sections for aCGH and to obtain results comparable to those from frozen tissues. Therefore, it is possible to perform aCGH analysis on archival small tissue biopsies and potentially histologically defined microscopic lesions, such as carcinoma in situ. With the advantage of genomic arrays, particularly its versatile design, high throughput and high resolution, application of aCGH on archival FFPE tissues, an immense resource of well-documented pathological specimens, will no doubt play a significant role in disease gene discovery and translational research.

\section{Acknowledgements}

We thank Martin McCabe for providing data partially used to generate the reference data set. The Du lab (M-Q Du, N Johnson and R Hamoudi) was supported by research grants from Leukaemia Research Fund, United Kingdom. The Collins Lab (V Peter Collins, K Ichimura, L Liu and D Pearson) was supported by research grants from Cancer Research UK.

\section{Duality of interest}

None declared.

\section{References}

1 Ishkanian AS, Malloff CA, Watson SK, et al. A tiling resolution DNA microarray with complete coverage of the human genome. Nat Genet 2004;36:299-303.

2 Fiegler H, Carr P, Douglas EJ, et al. DNA microarrays for comparative genomic hybridisation based on DOPPCR amplification of BAC and PAC clones. Genes Chromosomes Cancer 2003;36:361-374.

3 Seng TJ, Ichimura K, Liu L, et al. Complex chromosome 22 rearrangements in astrocytic tumors identified using microsatellite and chromosome 22 tile path array analysis. Genes Chromosomes Cancer 2005;43: 181-193.

4 Ichimura K, Mungall AJ, Fiegler H, et al. Small regions of overlapping deletions on 6q26 in human astrocytic tumors identified using chromosome 6 tile path arrayCGH. Oncogene 2006;25:1261-1271.

5 Gribble SM, Fiegler H, Burford DC, et al. Applications of combined DNA microarray and chromosome sorting technologies. Chromosome Res 2004;12:35-43.

6 Nessling M, Richter K, Schwaenen C, et al. Candidate genes in breast cancer revealed by microarray-based comparative genomic hybridization of archived tissue. Cancer Res 2005;65:439-447. 
7 Harvell JD, Kohler S, Zhu S, et al. High-resolution array-based comparative genomic hybridization for distinguishing paraffin-embedded Spitz nevi and melanomas. Diagnost Mol Pathol 2004;13:22-25.

8 Ried T, Just K, Holtgreve-Grez H, et al. Comparative genomic hybridization of formalin-fixed, paraffinembedded breast tumors reveals different patterns of chromosomal gains and losses in fibroadenomas and diploid and aneuploid carcinomas. Cancer Res 1995; 55:5415-5423.

9 Loo LWM, Grove DI, Williams EM, et al. Array comparative genomic hybridization analysis of genomic alterations in breast cancer subtypes. Cancer Res 2004;64:8541-8549.

10 Bredel M, Bredel C, Juric D, et al. Amplification of whole tumor genomes and gene-by-gene mapping of genomic aberrations from limited sources of freshfrozen and paraffin-embedded DNA. J Mol Diagn 2005; 7:171-182.

11 Little SE, Vuononvirta R, Reis-Filho JS, et al. Array $\mathrm{CGH}$ using whole genome amplification of freshfrozen and formalin-fixed, paraffin-embedded tumor DNA. Genomics 2006;87:298-306.

12 Liu D, Liu C, DeVries S, et al. LM-PCR permits highly representative whole genome amplification of DNA isolated from small number of cells and paraffinembedded tumor tissue sections. Diagnost Mol Pathol 2004;13:105-115.
13 Ichimura K, Schmidt EE, Goike HM, et al. Human glioblastomas with no alterations of the CDKN2A (p16INK4A, MTS1) and CDK4 genes have frequent mutations of the retinoblastoma gene. Oncogene 1996; 13:1065-1072.

14 van Dongen JJ, Langerak AW, Bruggeman M, et al. Design and standardization of PCR primers and protocols for detection of clonal immunoglobulin and T-cell receptor gene recombinations in suspect lymphoproliferations: report of the BIOMED-2 Concerted Action BMH4-CT98-3936. Leukemia 2003;17:2257-2317.

15 McCabe MG, Ichimura K, Liu L, et al. High resolution array-based comparative genomic hybridisation of medulloblastomas and supra-tentorial primitive neuroectodermal tumors. J Neuropathol Exp Neurol 2006 (in press).

16 Chen W, Houldsworth J, Olshen AB, et al. Array comparative genomic hybridization reveals genomic copy number changes associated with outcome in diffuse large B-cell lymphomas. Blood 2005;107: 2477-2485

17 R Development Core Team. R: A language and environment for statistical computing. $\mathrm{R}$ Foundation for statistical computing: Vienna, Austria.

18 Liu J, Zabarovska VI, Braga E, et al. Loss of heterozygosity in tumor cells requires re-evaluation: the data are biased by the size-dependent differential sensitivity of allele detection. FEBS Lett 1999;462:121-128.

Supplementary Information accompanies the paper on the Laboratory Investigation website (http:// www.nature.com/labinvest) 